\title{
Characterizations of weakly compact sets and new fixed point free maps in $c_{0}$
}

\author{
by \\ P. N. Dowling (Oxford, OH), C. J. Lennard (Pittsburgh, PA) \\ and B. TuretT (Rochester, MI)
}

\begin{abstract}
We give a basic sequence characterization of relative weak compactness in $c_{0}$ and we construct new examples of closed, bounded, convex subsets of $c_{0}$ failing the fixed point property for nonexpansive self-maps. Combining these results, we derive the following characterization of weak compactness for closed, bounded, convex subsets $C$ of $c_{0}$ : such a $C$ is weakly compact if and only if all of its closed, convex, nonempty subsets have the fixed point property for nonexpansive mappings.
\end{abstract}

1. Introduction. While the main result of this paper is a characterization of weak compactness in the Banach space $c_{0}$, this work has its origins in metric fixed point theory. In his celebrated paper of 1981, Maurey [9] used ultrapower techniques to prove a number of very interesting results in metric fixed point theory. One result of particular interest is that closed bounded convex nonempty subsets of $c_{0}$ that are weakly compact have the fixed point property for nonexpansive self-maps. Considerable effort had been expended in the years before 1980 to prove this result and some partial results were known; most notably the results of Haydon, Odell and Sternfeld [6], and Odell and Sternfeld [10]. In the years immediately following the appearance of Maurey's paper, several people worked on extending Maurey's result in $c_{0}$ to a larger class of Banach spaces. Borwein and Sims [2] proved an analogue of Maurey's result in the setting of Banach lattices under some technical conditions, and Lin [7] proved an analogue of Maurey's result in the Banach spaces with a 1-unconditional basis.

In recent years, the converse of Maurey's result has been a topic of active research. Llorens-Fuster and Sims [8] proved that certain closed bounded

2000 Mathematics Subject Classification: 47H10, 47H09, 46B50, 46B45.

The second author thanks Paddy Dowling and the Department of Mathematics and Statistics at Miami University for their hospitality during the preparation of this paper; as well as the Analysis seminar members for helpful comments and suggestions. He also acknowledges the financial support of Miami University.

The third author thanks Professors L. Weis and A. Kirsch for their hospitality and Universität Karlsruhe for financial support during the preparation of this paper. 
convex nonempty subsets of $c_{0}$ which are not weakly compact but are "close to weakly compact" fail the fixed point property for nonexpansive mappings. These examples led them to conjecture that closed bounded convex nonempty subsets of $c_{0}$ have the fixed point property if and only if they are weakly compact. In this paper we prove a result that partially supports this conjecture of Llorens-Fuster and Sims. Specifically, we prove that a closed bounded convex nonempty subset of $c_{0}$ is weakly compact if and only if all of its closed convex nonempty subsets have the fixed point property. As a consequence, we also prove the result analogous to this in the space $\left(c,\|\cdot\|_{\infty}\right)$ of convergent scalar sequences.

The key to proving the results mentioned above is to build, inside any closed bounded convex subset of $c_{0}$, a sequence which acts very much like the usual summing basis in $c_{0}$. We will construct nonexpansive fixed point free mappings on the closed convex hull of this sequence. One consequence of our construction is that a subset $C$ of $c_{0}$ is relatively weakly compact if and only if $C$ is bounded and the convex hull of $C$ does not contain an asymptotically isometric copy of the summing basis. Our construction can be further modified to prove that a subset of $c_{0}$ is relatively weakly compact if and only if it is bounded and does not contain a copy of the summing basis.

We note here that all our results hold independently of whether the underlying scalar field is the real numbers $\mathbb{R}$ or the complex numbers $\mathbb{C}$.

Finally, we remark that, working independently of each other, we and Domínguez Benavides, Japón Pineda and Prus [4, Proposition 3.1(c), Theorem 3.2(c) and Corollary 3.4(c)] have both recently proven related results for $c_{0}$. It turns out that our results are more general than those of Domínguez Benavides, Japón Pineda and Prus. They prove that a closed bounded convex nonempty subset $C$ of $c_{0}$ is weakly compact if and only if there exists a constant $M>1$ such that all of $C$ 's closed convex nonempty subsets have the fixed point property for affine mappings which are uniformly Lipschitzian with the constant $M$. (In [4] an analogue of this result is also proven for $c_{0}(\Gamma)$, for any infinite set $\Gamma$, and for the James space $J_{p}$.)

Note that [4] employs a right shift mapping $T$ in Theorem 3.2 and Corollary 3.4 there. We use the averaging of iterated right shift operators, as described in Theorem 2 below. This is one of the main new tools of our paper. These tools enable us to reach the stronger conclusion mentioned above.

2. The results. We begin this section with a definition of asymptotically isometric $c_{0}$-summing basic sequence. Throughout this paper $c_{00}$ will denote the space of finitely nonzero sequences. 
Definition 1. Let $\left(x_{n}\right)_{n \in \mathbb{N}}$ be a sequence in a Banach space $X$. We say that $\left(x_{n}\right)$ is an asymptotically isometric $c_{0}$-summing basic sequence if there exists a null sequence $\left(\varepsilon_{n}\right)$ in $(0, \infty)$ such that for all $\left(t_{n}\right)_{n} \in c_{00}$,

$$
\sup _{n \geq 1}\left(\frac{1}{1+\varepsilon_{n}}\right)\left|\sum_{j=n}^{\infty} t_{j}\right| \leq\left\|\sum_{n=1}^{\infty} t_{n} x_{n}\right\| \leq \sup _{n \geq 1}\left(1+\varepsilon_{n}\right)\left|\sum_{j=n}^{\infty} t_{j}\right| .
$$

Note that we may replace $c_{00}$ by $\ell_{1}$ in the above definition. If $L>0$, we will refer to a sequence $\left(x_{n}\right)$ as an $L$-scaled asymptotically isometric $c_{0}$-summing basic sequence if the sequence $\left(x_{n} / L\right)$ is an asymptotically isometric $c_{0}$-summing basic sequence.

Theorem 2. Let $X$ be a Banach space with a norm $\|\cdot\|$, and let $K$ be a closed bounded convex subset of $X$. Let $\left(\varepsilon_{n}\right)$ be a null sequence in $(0, \infty)$ with $\varepsilon_{n}<2^{-1} 4^{-n}$ for all $n \geq 2$. If $K$ contains a sequence $\left(x_{n}\right)_{n \in \mathbb{N}}$ such that $(\dagger)$ holds for all $\left(t_{n}\right)_{n} \in c_{00}$, then $K$ contains a nonempty closed convex subset $C$ for which there is a nonexpansive affine mapping $T: C \rightarrow C$ that fails to have a fixed point in $C$. Moreover, $T$ is contractive, i.e. $\|T(x)-T(y)\|<$ $\|x-y\|$ for all $x, y \in C$ with $x \neq y$.

Proof. Define $C=\overline{\mathrm{CO}}\left\{x_{n}: n \in \mathbb{N}\right\}$, the closed convex hull of the sequence $\left(x_{n}\right)$. It is easy to check that we also have

$$
C=\left\{\sum_{n=1}^{\infty} t_{n} x_{n}: t_{n} \geq 0 \text { for all } n \in \mathbb{N} \text { and } \sum_{n=1}^{\infty} t_{n}=1\right\} .
$$

Now, define $T\left(x_{n}\right)=\sum_{j=1}^{\infty} 2^{-j} x_{j+n}$ for all $n \in \mathbb{N}$, and extend $T$ linearly to $C$; i.e.

$$
T(x)=\sum_{n=1}^{\infty} t_{n} T\left(x_{n}\right) \quad \text { for all } x=\sum_{n=1}^{\infty} t_{n} x_{n} \in C .
$$

It is clear that $T$ maps $C$ into $C$. Further, $T$ has no fixed point in $C$. Indeed, fix $x \in C$ as above and suppose that $T(x)=x$. Now, $x=\sum_{n=1}^{\infty} t_{n} x_{n}$ implies that

$$
\begin{aligned}
T(x) & =\sum_{k \in \mathbb{N}} t_{k} T\left(x_{k}\right)=\sum_{k \in \mathbb{N}} t_{k} \sum_{j \in \mathbb{N}} \frac{1}{2^{j}} x_{j+k} \\
& =\sum_{k \in \mathbb{N}} \sum_{j \in \mathbb{N}} \frac{1}{2^{j}} t_{k} x_{j+k}=\sum_{m \geq 2}\left(\sum_{j, k \in \mathbb{N}: j+k=m} \frac{1}{2^{j}} t_{k}\right) x_{m} .
\end{aligned}
$$

Thus, $T(x)=\sum_{m=1}^{\infty} s_{m} x_{m}$, where $s_{1}=0$ and for all $m \geq 2$,

$$
s_{m}=\sum_{j, k \in \mathbb{N}: j+k=m} \frac{1}{2^{j}} t_{k}=\frac{1}{2^{m-1}} t_{1}+\ldots+\frac{1}{2^{1}} t_{m-1} .
$$

But $x=T(x)$. It follows that $t_{1}=s_{1}=0, t_{2}=(1 / 2) t_{1}=0, t_{3}=\left(1 / 2^{2}\right) t_{1}+$ $(1 / 2) t_{2}=0, \ldots$; and therefore each $t_{n}=0$, by induction. This contradicts the fact that $\sum_{n=1}^{\infty} t_{n}=1$. So, $T$ is fixed point free on $C$. 
We will complete the proof by showing that $T$ is contractive on $C$. Let $x=\sum_{n=1}^{\infty} t_{n} x_{n}$ and $y=\sum_{n=1}^{\infty} s_{n} x_{n}$, where $t_{n}, s_{n} \geq 0$ for all $n \in \mathbb{N}$ and $\sum_{n=1}^{\infty} t_{n}=\sum_{n=1}^{\infty} s_{n}=1$. Also suppose that $x \neq y$. Then

$$
T(x)-T(y)=\sum_{n=1}^{\infty} t_{n} T\left(x_{n}\right)-\sum_{n=1}^{\infty} s_{n} T\left(x_{n}\right)=\sum_{n=1}^{\infty}\left(t_{n}-s_{n}\right) T\left(x_{n}\right) .
$$

Let $\alpha_{n}=t_{n}-s_{n}$ for all $n \in \mathbb{N}$ and note that $\sum_{n=1}^{\infty} \alpha_{n}=0$. Similarly to an argument given above, it follows that

$$
\begin{aligned}
T(x)-T(y) & =\sum_{k=1}^{\infty} \alpha_{k} T\left(x_{k}\right)=\sum_{m=2}^{\infty}\left(\sum_{j, k \in \mathbb{N}: j+k=m} \frac{1}{2^{j}} \alpha_{k}\right) x_{m} \\
& =\sum_{n=1}^{\infty}\left(\frac{1}{2^{n}} \alpha_{1}+\frac{1}{2^{n-1}} \alpha_{2}+\frac{1}{2^{n-2}} \alpha_{3}+\ldots+\frac{1}{2} \alpha_{n}\right) x_{n+1}
\end{aligned}
$$

$(n=m-1)$. Therefore,

$$
\begin{aligned}
\| & T(x)-T(y) \| \leq \sup _{m \geq 1}\left(1+\varepsilon_{m}\right)\left|\sum_{n=m}^{\infty}\left(\sum_{j, k \in \mathbb{N}: j+k=n} \frac{1}{2^{j}} \alpha_{k}\right)\right| \\
= & \left(1+\varepsilon_{1}\right)\left|0+\left(\frac{1}{2} \alpha_{1}\right)+\left(\frac{1}{2^{2}} \alpha_{1}+\frac{1}{2} \alpha_{2}\right)+\left(\frac{1}{2^{3}} \alpha_{1}+\frac{1}{2^{2}} \alpha_{2}+\frac{1}{2} \alpha_{3}\right)+\ldots\right| \\
& \vee\left(1+\varepsilon_{2}\right)\left|\left(\frac{1}{2} \alpha_{1}\right)+\left(\frac{1}{2^{2}} \alpha_{1}+\frac{1}{2} \alpha_{2}\right)+\left(\frac{1}{2^{3}} \alpha_{1}+\frac{1}{2^{2}} \alpha_{2}+\frac{1}{2} \alpha_{3}\right)+\ldots\right| \\
& \vee\left(1+\varepsilon_{3}\right) \mid\left(\frac{1}{2^{2}} \alpha_{1}+\frac{1}{2} \alpha_{2}\right)+\left(\frac{1}{2^{3}} \alpha_{1}+\frac{1}{2^{2}} \alpha_{2}+\frac{1}{2} \alpha_{3}\right) \\
& +\left(\frac{1}{2^{4}} \alpha_{1}+\frac{1}{2^{3}} \alpha_{2}+\frac{1}{2^{2}} \alpha_{3}+\frac{1}{2} \alpha_{4}\right)+\ldots \mid \vee \ldots \\
= & \sup _{n \geq 3}\left(1+\varepsilon_{n}\right)\left|\frac{1}{2^{n-2}} \sum_{j=2}^{\infty} \alpha_{j}+\frac{1}{2^{n-3}} \sum_{j=3}^{\infty} \alpha_{j}+\ldots+\frac{1}{2^{1}} \sum_{j=n-1}^{\infty} \alpha_{j}\right| \\
\leq & \sup _{n \geq 3}\left(1+\varepsilon_{n}\right)\left(\frac{1}{2^{n-2}}\left|\sum_{j=2}^{\infty} \alpha_{j}\right|+\frac{1}{2^{n-3}}\left|\sum_{j=3}^{\infty} \alpha_{j}\right|+\ldots+\frac{1}{2^{1}}\left|\sum_{j=n-1}^{\infty} \alpha_{j}\right|\right) \\
= & \sup _{n \geq 3}\left(1+\varepsilon_{n}\right)\left(\frac{1+2 \varepsilon_{2}}{2^{n-2}} \frac{1}{1+2 \varepsilon_{2}}\left|\sum_{j=2}^{\infty} \alpha_{j}\right|+\frac{1+2 \varepsilon_{3}}{2^{n-3}} \frac{1}{1+2 \varepsilon_{3}}\left|\sum_{j=3}^{\infty} \alpha_{j}\right|+\ldots\right. \\
& \left.+\frac{1+2 \varepsilon_{n-1}}{2^{1}} \frac{1}{1+2 \varepsilon_{n-1}}\left|\sum_{j=n-1}^{\infty} \alpha_{j}\right|\right) \\
\leq & \max _{m \geq 1} \frac{1}{1+2 \varepsilon_{m}}\left|\sum_{j=m}^{\infty} \alpha_{j}\right| \cdot Q, \\
& \quad Q \quad
\end{aligned}
$$


where

$$
\begin{aligned}
Q:= & \sup _{n \geq 3}\left(1+\varepsilon_{n}\right)\left(\frac{1+2 \varepsilon_{2}}{2^{n-2}}+\frac{1+2 \varepsilon_{3}}{2^{n-3}}+\ldots+\frac{1+2 \varepsilon_{n-1}}{2^{1}}\right) \\
\leq & \sup _{n \geq 3}\left(1+\frac{1}{2 \cdot 4^{n}}\right)\left(\left[\frac{1}{2^{n-2}}+\frac{1}{2^{n-3}}+\ldots+\frac{1}{2^{1}}\right]\right. \\
& \left.+\left[\frac{1}{4^{2} 2^{n-2}}+\frac{1}{4^{3} 2^{n-3}}+\ldots+\frac{1}{4^{n-1} 2^{1}}\right]\right) \\
= & \sup _{n \geq 3}\left(1+\frac{1}{2 \cdot 4^{n}}\right)\left(1-\frac{1}{2^{n-2}}+\left[\frac{1}{2^{n+2}}+\frac{1}{2^{n+3}}+\ldots+\frac{1}{2^{2 n-1}}\right]\right) \\
\leq & \sup _{n \geq 3}\left(1+\frac{1}{4^{n}}\right)\left(1-\frac{1}{2^{n-2}}+\frac{1}{2^{n+1}}\right) \leq \sup _{n \geq 3}\left(1+\frac{1}{4^{n}}-\frac{7}{2^{n+1}}\right)=1 .
\end{aligned}
$$

Recall that $x \neq y$. So, $t_{i} \neq s_{i}$ for some $i$, and so some $\alpha_{i} \neq 0$. Thus, $\sum_{j=m}^{\infty} \alpha_{j} \neq 0$ for some $m$. Consequently,

$$
\begin{aligned}
\|T(x)-T(y)\| & \leq \max _{m \geq 1} \frac{1}{1+2 \varepsilon_{m}}\left|\sum_{j=m}^{\infty} \alpha_{j}\right|<\max _{m \geq 1} \frac{1}{1+\varepsilon_{m}}\left|\sum_{j=m}^{\infty} \alpha_{j}\right| \\
& \leq\left\|\sum_{j=1}^{\infty} \alpha_{j} x_{j}\right\|
\end{aligned}
$$

and therefore $\|T(x)-T(y)\|<\|x-y\|$ for all $x, y \in C$ with $x \neq y$.

Next, note that a subsequence of an asymptotically isometric $c_{0}$-summing basic sequence is again an asymptotically isometric $c_{0}$-summing basic sequence. Moreover, we can choose the subsequence in such a way that the corresponding new sequence of $\varepsilon_{n}$ 's, $\left(\widehat{\varepsilon}_{n}\right)_{n \geq 1}$ say, satisfies $\widehat{\varepsilon}_{n}<2^{-1} 4^{-n}$ for all $n \geq 2$. Hence, we immediately have the following corollary of the last result:

Corollary 3. If $L>0$ and $K$ is a closed bounded convex subset of a Banach space that contains an L-scaled asymptotically isometric $c_{0^{-}}$ summing basic sequence, then $K$ contains a nonempty closed convex subset $C$ such that there is a nonexpansive affine mapping $T: C \rightarrow C$ which fails to have a fixed point in $C$.

REMARK. Now that we have a method for recognizing whether a closed bounded convex subset of a Banach space contains a closed bounded convex subset that will support a fixed point free nonexpansive mapping, we will show that closed bounded convex subsets of $\left(c_{0},\|\cdot\|_{\infty}\right)$ that are not weakly compact are examples of such sets. Most of our work here will involve showing that a certain sequence is an asymptotically isometric $c_{0^{-}}$ summing basic sequence. Note that if a sequence $\left(x_{n}\right)$ is an asymptotically 
isometric $c_{0}$-summing basic sequence and if we define a sequence $\left(w_{n}\right)$ by $w_{n}:=x_{n}-x_{n-1}$, where $x_{0}:=0$, then we have

$$
\sup _{n}\left(\frac{1}{1+\varepsilon_{n}}\right)\left|t_{n}\right| \leq\left\|\sum_{n=1}^{\infty} t_{n} w_{n}\right\| \leq \sup _{n}\left(1+\varepsilon_{n}\right)\left|t_{n}\right|
$$

for all $\left(t_{n}\right) \in c_{0}$. This condition is very close to saying that the sequence is an asymptotically isometric $c_{0}$-sequence in the sense of [5]. Conversely, we note that if the sequence $\left(w_{n}\right)$ defined as above satisfies condition $(*)$, then the sequence $\left(x_{n}\right)$ is an asymptotically isometric $c_{0}$-summing basic sequence.

Theorem 4 below is an "asymptotically isometric" analogue of Proposition 3.1 and part of Theorem 3.2 of [4], which are " $(1+\varepsilon)$-isomorphic" in flavour.

TheOrem 4 . Let $K$ be a closed bounded convex subset of $\left(c_{0},\|\cdot\|_{\infty}\right)$ which is not weakly compact. Then $K$ contains an L-scaled asymptotically isometric $c_{0}$-summing basic sequence, for some $L>0$. Consequently, $K$ contains a closed convex nonempty subset $C$ that fails the fixed point property for some nonexpansive mapping $T$ on $C$. Further, we may choose $T$ to be affine and contractive.

Proof. Since $K$ is not weakly compact, by the Eberlein-Shmul'yan theorem and the weak* sequential compactness of the closed unit ball in $\ell_{\infty}$, there exists a sequence $\left(x_{n}\right)_{n \in \mathbb{N}}$ in $K$ which converges weak* to an element $x=\left(\xi_{i}\right)_{i \in \mathbb{N}}$ in $\ell_{\infty} \backslash c_{0}$. Similarly, we denote each $x_{n}$ by $\left(\xi_{i}^{n}\right)_{i \in \mathbb{N}}=\sum_{i=1}^{\infty} \xi_{i}^{n} e_{i}$, where $\left(e_{i}\right)_{i \in \mathbb{N}}$ is the canonical unit vector basis of $c_{0}$.

Let $L=\lim \sup _{i}\left|\xi_{i}\right|$. Clearly, $L>0$ and so by scaling the set $K$ and the sequence $\left(x_{n}\right)$ by $L$, we can assume that $L=1$.

Fix $\delta$ with $0<\delta<4^{-7}$.

Let $N_{0}=0$ and choose $n_{1}=1$. Since $x_{n_{1}} \in c_{0}$ and $\limsup _{i}\left|\xi_{i}\right|=1$, we may choose $N_{1} \geq 1$ so that $\left|\xi_{i}^{n_{1}}\right|<\delta / 4^{1+2}$ for all $i \geq N_{1}, \max _{1 \leq i \leq N_{1}}\left|\xi_{i}\right|>$ $1-\delta / 4^{1+2}$ and $\sup _{i>N_{1}}\left|\xi_{i}\right|<1+\delta / 4^{1+2}$. Because $x_{n} \rightarrow x$ coordinatewise, we may choose $n_{2}>n_{1}$ so that $\left|\xi_{i}^{n_{2}}-\xi_{i}\right|<\delta / 4^{2+2}$ for all $1 \leq i \leq N_{1}$. Since $x_{n_{1}}, x_{n_{2}} \in c_{0}$ and $\lim \sup _{i}\left|\xi_{i}\right|=1$, we can choose $N_{2}>N_{1}$ so that $\left|\xi_{i}^{n_{1}}\right|+\left|\xi_{i}^{n_{2}}\right|<\delta / 4^{2+2}$ for all $i \geq N_{2}, \max _{N_{1}<i \leq N_{2}}\left|\xi_{i}\right|>1-\delta / 4^{2+2}$ and $\sup _{i>N_{2}}\left|\xi_{i}\right|<1+\delta / 4^{2+2}$.

Next, choose $n_{3}>n_{2}$ so that $\left|\xi_{i}^{n_{3}}-\xi_{i}\right|<\delta / 4^{3+2}$ for all $1 \leq i \leq N_{2}$. Choose $N_{3}>N_{2}$ so that $\left|\xi_{i}^{n_{1}}\right|+\left|\xi_{i}^{n_{2}}\right|+\left|\xi_{i}^{n_{3}}\right|<\delta / 4^{3+2}$ for all $i \geq N_{3}$, $\max _{N_{2}<i \leq N_{3}}\left|\xi_{i}\right|>1-\delta / 4^{3+2}$ and $\sup _{i>N_{3}}\left|\xi_{i}\right|<1+\delta / 4^{3+2}$.

Continuing inductively in this manner we produce integer sequences $1 \leq$ $n_{1}<n_{2}<\ldots$ and $1 \leq N_{1}<N_{2}<\ldots$ so that 
(1) $\left|\xi_{i}^{n_{k}}-\xi_{i}\right|<\delta / 4^{k+2}$ for all $1 \leq i \leq N_{k-1}$ and all $k \geq 2$;

(2) $\max _{N_{k-1}<i \leq N_{k}}\left|\xi_{i}\right|>1-\delta / 4^{k+2}$ for all $k \in \mathbb{N}$;

(3) $\sup _{i>N_{k}}\left|\xi_{i}\right|<1+\delta / 4^{k+2}$ for all $k \in \mathbb{N}$; and

(4) $\sum_{l=1}^{k}\left|\xi_{i}^{n_{l}}\right|<\delta / 4^{k+2}$ for all $i \geq N_{k}$ and all $k \in \mathbb{N}$.

To simplify our notation we now relabel $x_{n_{k}}$ by $x_{k}$. Thus conditions (1)-(4) become

$\left(1^{\prime}\right)\left|\xi_{i}^{k}-\xi_{i}\right|<\delta / 4^{k+2}$ for all $1 \leq i \leq N_{k-1}$ and all $k \geq 2$;

$\left(2^{\prime}\right) \max _{N_{k-1}<i \leq N_{k}}\left|\xi_{i}\right|>1-\delta / 4^{k+2}$ for all $k \in \mathbb{N}$;

$\left(3^{\prime}\right) \sup _{i>N_{k}}\left|\xi_{i}\right|<1+\delta / 4^{k+2}$ for all $k \in \mathbb{N}$; and

$\left(4^{\prime}\right) \sum_{l=1}^{k}\left|\xi_{i}^{l}\right|<\delta / 4^{k+2}$ for all $i \geq N_{k}$ and all $k \in \mathbb{N}$.

At this stage we have some control of $\xi_{i}^{k}$ when $1 \leq i \leq N_{k-1}$ and when $i \geq N_{k}$ (for $k \geq 2$ ). To gain some control when $N_{k-1}<i \leq N_{k}$, we will average the $x_{k}$ 's.

Since $K$ is bounded, there exists a constant $B>0$ so that $\left\|x_{k}\right\| \leq B$ for all $k \in \mathbb{N}$. Fix a strictly decreasing sequence $\left(\delta_{n}\right)$ in $(0,1)$ so that $\delta_{n}<\delta / 4^{n}$ for all $n \in \mathbb{N}$. Choose a strictly increasing sequence $(M(n))_{n \in \mathbb{N}}$ in $\mathbb{N}$ so that

$$
B / \Delta_{n}<\delta_{n} \quad \text { for all } n \in \mathbb{N},
$$

where

$$
\Delta_{n}=M(n)-M(n-1) \text { and } M(0)=0 .
$$

For each $n \in \mathbb{N}$, define

$$
y_{n}:=\frac{1}{\Delta_{n}} \sum_{l=M(n-1)+1}^{M(n)} x_{l} .
$$

Denote $y_{n}$ by $\sum_{i=1}^{\infty} y_{i}^{n} e_{i}$ and note that

$$
y_{i}^{n}=\frac{1}{\Delta_{n}} \sum_{l=M(n-1)+1}^{M(n)} \xi_{i}^{l} \quad \text { for all } i \in \mathbb{N} \text { and } n \in \mathbb{N} .
$$

We now need to estimate the size of each $y_{i}^{n}$. For this we consider three separate cases.

Case 1. If $i>N_{M(n)}$, then by $\left(4^{\prime}\right)$ above,

$$
\left|y_{i}^{n}\right| \leq \frac{1}{\Delta_{n}} \sum_{l=M(n-1)+1}^{M(n)}\left|\xi_{i}^{l}\right| \leq \frac{1}{\Delta_{n}} \sum_{l=1}^{M(n)}\left|\xi_{i}^{l}\right|<\frac{1}{\Delta_{n}} \frac{\delta}{4^{M(n)+2}} \leq \frac{\delta}{4^{n+2}} .
$$


CASE 2. If $i \leq N_{M(n-1)}$ and $n \geq 2$, then by $\left(1^{\prime}\right)$,

$$
\begin{aligned}
\left|y_{i}^{n}-\xi_{i}\right| & \leq \frac{1}{\Delta_{n}} \sum_{l=M(n-1)+1}^{M(n)}\left|\xi_{i}^{l}-\xi_{i}\right| \\
& <\frac{1}{\Delta_{n}} \sum_{l=M(n-1)+1}^{M(n)} \frac{\delta}{4^{M(n-1)+1+2}}=\frac{\delta}{4^{M(n-1)+3}} \leq \frac{\delta}{4^{n+2}} .
\end{aligned}
$$

CASE 3. If $N_{M(n-1)}<i \leq N_{M(n)}$, then there exists a unique $j \in \mathbb{N}$ with $1 \leq j \leq \Delta_{n}$ so that $N_{M(n-1)+j-1}<i \leq N_{M(n-1)+j}$. Then, via $\left(4^{\prime}\right)$ and $\left(1^{\prime}\right)$, we have

$$
\begin{aligned}
\mid y_{i}^{n}- & \left(\frac{\Delta_{n}-j}{\Delta_{n}}\right) \xi_{i}\left|=\frac{1}{\Delta_{n}}\right| \sum_{l=M(n-1)+1}^{M(n)} \xi_{i}^{l}-\left(\Delta_{n}-j\right) \xi_{i} \mid \\
& \leq \frac{1}{\Delta_{n}}\left\{\sum_{l=M(n-1)+1}^{M(n-1)+j-1}\left|\xi_{i}^{l}\right|+\left|\xi_{i}^{M(n-1)+j}\right|+\sum_{l=M(n-1)+j+1}^{M(n)}\left|\xi_{i}^{l}-\xi_{i}\right|\right\}
\end{aligned}
$$

(where the first sum is 0 if $j=1$ and the last sum is 0 if $j=\Delta_{n}$ )

$$
\begin{aligned}
& \leq \frac{1}{\Delta_{n}}\left\{\frac{\delta}{4^{M(n-1)+j+1}}+B+\sum_{l=M(n-1)+j+1}^{M(n)} \frac{\delta}{4^{M(n-1)+j+3}}\right\} \\
& \leq \frac{\delta}{4^{n+1}}+\frac{B}{\Delta_{n}}+\frac{\delta}{4^{n+3}}<\frac{\delta}{4^{n+1}}+\delta_{n}+\frac{\delta}{4^{n+3}}<\frac{\delta}{4^{n}}+\delta_{n}<\frac{\delta}{4^{n-1}} .
\end{aligned}
$$

We now wish to show that the sequence $\left(y_{n}\right)_{n \in \mathbb{N}}$ is an asymptotically isometric $c_{0}$-summing basic sequence. By our remarks preceding this theorem, it suffices to show that $\left(w_{n}\right)_{n \in \mathbb{N}}$ satisfies condition $(*)$, where $w_{n}:=$ $y_{n}-y_{n-1}$, with $y_{0}:=0$. To this end, fix an element $\left(t_{k}\right)_{k \in \mathbb{N}}$ of $c_{00}$, and define $\sigma:=\sum_{k=1}^{\infty} t_{k} w_{k}$. Then for all $k \in \mathbb{N}$ we have

$$
w_{k}=\sum_{i \in \mathbb{N}} w_{i}^{k} e_{i}, \quad \text { where each } w_{i}^{k}:=y_{i}^{k}-y_{i}^{k-1} \text {. }
$$

Further,

$$
\sigma=\sum_{i \in \mathbb{N}} \sigma_{i} e_{i}, \quad \text { where each } \sigma_{i}:=\sum_{k=1}^{\infty} t_{k} w_{i}^{k} .
$$

Note that $\sigma \in c_{0}$, and so

$$
\begin{aligned}
\left\|\sum_{k=1}^{\infty} t_{k} w_{k}\right\|_{\infty} & =\|\sigma\|_{\infty}=\max _{i \in \mathbb{N}}\left|\sigma_{i}\right|=\max _{n \in \mathbb{N}} \max _{N_{M(n-1)}<i \leq N_{M(n)}}\left|\sigma_{i}\right| \\
& =\max _{n \in \mathbb{N}} \max _{1 \leq j \leq \Delta_{n}}\left|\sigma_{i}\right| .
\end{aligned}
$$


We wish to estimate $\|\sigma\|_{\infty}$ from above and below, in order to establish the pair of inequalities $(*)$, for some null sequence $\left(\varepsilon_{n}\right)_{n \in \mathbb{N}}$ in $(0, \infty)$ to be determined.

Fix $n \in \mathbb{N}$ and fix $j \in\left\{1, \ldots, \Delta_{n}\right\}$. Next, fix $i \in \mathbb{N}$ with $N_{M(n-1)+j-1}<$ $i \leq N_{M(n-1)+j}$. Then

$$
\begin{aligned}
& \left|\sigma_{i}\right|=\left|\sum_{k=1}^{\infty} t_{k} w_{i}^{k}\right| \leq\left|\sum_{k=1}^{n-1} t_{k} w_{i}^{k}\right|+\left|t_{n} w_{i}^{n}\right|+\left|t_{n+1} w_{i}^{n+1}\right|+\left|\sum_{k=n+2}^{\infty} t_{k} w_{i}^{k}\right| \\
& \leq \sum_{k=1}^{n-1}\|t\|_{\infty}\left|y_{i}^{k}-y_{i}^{k-1}\right|+\left|t_{n}\right|\left|w_{i}^{n}\right|+\left|t_{n+1}\right|\left|w_{i}^{n+1}\right|+\sum_{k=n+2}^{\infty}\|t\|_{\infty}\left|y_{i}^{k}-y_{i}^{k-1}\right| \\
& \leq 2\|t\|_{\infty} \sum_{k=1}^{n-1}\left|y_{i}^{k}\right|+\left|t_{n}\right|\left|w_{i}^{n}\right|+\left|t_{n+1}\right|\left|w_{i}^{n+1}\right|+2\|t\|_{\infty} \sum_{k=n+1}^{\infty}\left|y_{i}^{k}-\xi_{i}\right| \\
& \leq 2\|t\|_{\infty} \sum_{l=1}^{M(n-1)}\left|\xi_{i}^{l}\right|+\left|t_{n}\right|\left|w_{i}^{n}\right|+\left|t_{n+1}\right|\left|w_{i}^{n+1}\right|+2\|t\|_{\infty} \sum_{k=n+1}^{\infty} \frac{\delta}{4^{k+2}} \quad\left[\text { by }\left(1^{\prime}\right)\right] \\
& \leq 2\|t\|_{\infty} \frac{\delta}{4^{M(n-1)+2}}+\left|t_{n}\right|\left|w_{i}^{n}\right|+\left|t_{n+1}\right|\left|w_{i}^{n+1}\right|+2\|t\|_{\infty} \frac{\delta}{4^{n+2}} \quad\left[\text { by }\left(4^{\prime}\right)\right] \\
& \leq \frac{\delta}{4^{n}}\|t\|_{\infty}+\left|t_{n}\right|\left|w_{i}^{n}\right|+\left|t_{n+1}\right|\left|w_{i}^{n+1}\right| .
\end{aligned}
$$

By the estimates in Cases 3 and 1 above, we also have

$$
\begin{aligned}
\left|w_{i}^{n}\right| & =\left|y_{i}^{n}-y_{i}^{n-1}\right| \leq\left|y_{i}^{n}-\frac{\Delta_{n}-j}{\Delta_{n}} \xi_{i}\right|+\frac{\Delta_{n}-j}{\Delta_{n}}\left|\xi_{i}\right|+\left|y_{i}^{n-1}\right| \\
& \leq \frac{\delta}{4^{n-1}}+\frac{\Delta_{n}-j}{\Delta_{n}}\left|\xi_{i}\right|+\frac{\delta}{4^{n+1}} \leq \frac{2 \delta}{4^{n-1}}+\frac{\Delta_{n}-j}{\Delta_{n}}\left|\xi_{i}\right| .
\end{aligned}
$$

Moreover, by Cases 2 and 3 above,

$$
\begin{aligned}
\left|w_{i}^{n+1}\right| & =\left|y_{i}^{n+1}-y_{i}^{n}\right| \leq\left|y_{i}^{n+1}-\xi_{i}\right|+\left|y_{i}^{n}-\xi_{i}\right| \\
& \leq\left|y_{i}^{n+1}-\xi_{i}\right|+\left|y_{i}^{n}-\frac{\Delta_{n}-j}{\Delta_{n}} \xi_{i}\right|+\frac{j}{\Delta_{n}}\left|\xi_{i}\right| \\
& \leq \frac{\delta}{4^{n+3}}+\frac{\delta}{4^{n-1}}+\frac{j}{\Delta_{n}}\left|\xi_{i}\right| \leq \frac{2 \delta}{4^{n-1}}+\frac{j}{\Delta_{n}}\left|\xi_{i}\right| .
\end{aligned}
$$

Therefore,

$$
\begin{aligned}
\left|\sigma_{i}\right| & =\left|\sum_{k=1}^{\infty} t_{k} w_{i}^{k}\right| \leq \frac{\delta}{4^{n}}\|t\|_{\infty}+\left|t_{n}\right|\left|w_{i}^{n}\right|+\left|t_{n+1}\right|\left|w_{i}^{n+1}\right| \\
& \leq \frac{\delta}{4^{n}}\|t\|_{\infty}+\left|t_{n}\right|\left\{\frac{2 \delta}{4^{n-1}}+\frac{\Delta_{n}-j}{\Delta_{n}}\left|\xi_{i}\right|\right\}+\left|t_{n+1}\right|\left\{\frac{2 \delta}{4^{n-1}}+\frac{j}{\Delta_{n}}\left|\xi_{i}\right|\right\}
\end{aligned}
$$




$$
\begin{aligned}
& \leq \frac{\delta}{4^{n-3}}\|t\|_{\infty}+\left|t_{n}\right| \frac{\Delta_{n}-j}{\Delta_{n}}\left|\xi_{i}\right|+\left|t_{n+1}\right| \frac{j}{\Delta_{n}}\left|\xi_{i}\right| \\
& \leq \frac{\delta}{4^{n-3}}\|t\|_{\infty}+\max \left\{\left|t_{n}\right|,\left|t_{n+1}\right|\right\}\left|\xi_{i}\right| .
\end{aligned}
$$

In summary, we have shown that for all $n \in \mathbb{N}$ and all $i \in \mathbb{N}$ such that $N_{M(n-1)}<i \leq N_{M(n)}$, we have

$$
\left|\sigma_{i}\right|=\left|\sum_{k=1}^{\infty} t_{k} w_{i}^{k}\right| \leq \frac{\delta}{4^{n-3}}\|t\|_{\infty}+\max \left\{\left|t_{n}\right|,\left|t_{n+1}\right|\right\}\left|\xi_{i}\right|
$$

and thus for all $n \geq 2$, via $\left(3^{\prime}\right)$ above, we see that

$$
\begin{aligned}
\max _{N_{M(n-1)}<i \leq N_{M(n)}}\left|\sigma_{i}\right| & \leq \frac{\delta}{4^{n-3}}\|t\|_{\infty}+\max \left\{\left|t_{n}\right|,\left|t_{n+1}\right|\right\} \max _{N_{M(n-1)}<i \leq N_{M(n)}}\left|\xi_{i}\right| \\
& \leq \frac{\delta}{4^{n-3}}\|t\|_{\infty}+\max \left\{\left|t_{n}\right|,\left|t_{n+1}\right|\right\}\left(1+\frac{\delta}{4^{n-1+2}}\right) \\
& \leq \frac{\delta}{4^{n-4}}\|t\|_{\infty}+\max \left\{\left|t_{n}\right|,\left|t_{n+1}\right|\right\} .
\end{aligned}
$$

For $n=1$ we have the following two inequalities (keeping in mind that $\left.M_{0}=0\right)$ :

$$
\max _{N_{1}<i \leq N_{M(1)}}\left|\sigma_{i}\right| \leq \frac{\delta}{4^{1-4}}\|t\|_{\infty}+\max \left\{\left|t_{1}\right|,\left|t_{2}\right|\right\},
$$

which is derived exactly as for the case $n \geq 2$, and

$$
\max _{0<i \leq N_{1}}\left|\sigma_{i}\right| \leq \frac{\delta}{4^{1-4}}\|t\|_{\infty}+B\left|t_{1}\right|
$$

This second inequality is similarly derived, by using inequality $(\diamond)$ above and the fact that $B / \Delta_{1}<\delta_{1}<\delta / 4^{1}$. Since $B \geq 1$, these two inequalities can be combined to obtain the single inequality

$$
\max _{0<i \leq N_{M(1)}}\left|\sigma_{i}\right| \leq \frac{\delta}{4^{1-4}}\|t\|_{\infty}+\max \left\{B\left|t_{1}\right|,\left|t_{2}\right|\right\}
$$

Therefore, for each $n \in \mathbb{N}$,

$$
\text { (d) } \max _{N_{M(n-1)}<i \leq N_{M(n)}}\left|\sigma_{i}\right| \leq \frac{\delta}{4^{n-4}}\|t\|_{\infty}+\max \left\{A_{n}\left|t_{n}\right|, A_{n+1}\left|t_{n+1}\right|\right\} \text {, }
$$

where $A_{1}:=B$ and $A_{n}:=1$ for all $n \geq 2$.

On the other hand, let us again fix $n \in \mathbb{N}$ and $i \in \mathbb{N}$ with $N_{M(n-1)}<i$ $\leq N_{M(n)}$. Then, by similar arguments to those above, we also have

$$
\begin{aligned}
\left|\sigma_{i}\right|=\left|\sum_{k=1}^{\infty} t_{k} w_{i}^{k}\right| & \geq\left|t_{n}\right|\left|w_{i}^{n}\right|-\left|t_{n+1}\right|\left|w_{i}^{n+1}\right|-\left|\sum_{k=1}^{n-1} t_{k} w_{i}^{k}\right|-\left|\sum_{k=n+2}^{\infty} t_{k} w_{i}^{k}\right| \\
& \geq\left|t_{n}\right|\left|w_{i}^{n}\right|-\left|t_{n+1}\right|\left|w_{i}^{n+1}\right|-\frac{\delta}{4^{n}}\|t\|_{\infty} .
\end{aligned}
$$


In particular, for all $n \in \mathbb{N}$, if $i \in \mathbb{N}$ satisfies $N_{M(n-1)}<i \leq N_{M(n-1)+1}$, then

$$
\begin{aligned}
\left|\sigma_{i}\right| \geq & \left|t_{n}\right|\left|y_{i}^{n}-y_{i}^{n-1}\right|-\left|t_{n+1}\right|\left|y_{i}^{n+1}-y_{i}^{n}\right|-\frac{\delta}{4^{n}}\|t\|_{\infty} \\
\geq & \left|t_{n}\right|\left[\left|\xi_{i}\right|-\left|y_{i}^{n}-\xi_{i}\right|-\left|y_{i}^{n-1}\right|\right]-\left|t_{n+1}\right|\left[\left|y_{i}^{n+1}-\xi_{i}\right|+\left|y_{i}^{n}-\xi_{i}\right|\right]-\frac{\delta}{4^{n}}\|t\|_{\infty} \\
\geq & \left|t_{n}\right|\left[\left|\xi_{i}\right|-\left|y_{i}^{n}-\frac{\Delta_{n}-1}{\Delta_{n}} \xi_{i}\right|-\frac{1}{\Delta_{n}}\left|\xi_{i}\right|-\left|y_{i}^{n-1}\right|\right] \\
& \quad-\left|t_{n+1}\right|\left[\left|y_{i}^{n+1}-\xi_{i}\right|+\left|y_{i}^{n}-\frac{\Delta_{n}-1}{\Delta_{n}} \xi_{i}\right|+\frac{1}{\Delta_{n}}\left|\xi_{i}\right|\right]-\frac{\delta}{4^{n}}\|t\|_{\infty} \\
\geq & \left|t_{n}\right|\left[\left|\xi_{i}\right|-\frac{\delta}{4^{n-1}}-\frac{B}{\Delta_{n}}-\frac{\delta}{4^{n+1}}\right]-\left|t_{n+1}\right|\left[\frac{\delta}{4^{n+3}}+\frac{\delta}{4^{n-1}}+\frac{B}{\Delta_{n}}\right]-\frac{\delta}{4^{n}}\|t\|_{\infty}
\end{aligned}
$$

(by Cases 3, 1, 2 and 3 respectively, and the fact that each $\left|\xi_{i}\right| \leq B$ )

$$
\begin{aligned}
& \geq\left|t_{n}\right|\left|\xi_{i}\right|-\|t\|_{\infty}\left[\frac{\delta}{4^{n-1}}+\delta_{n}+\frac{\delta}{4^{n+1}}\right] \\
& \quad-\|t\|_{\infty}\left[\frac{\delta}{4^{n+3}}+\frac{\delta}{4^{n-1}}+\delta_{n}\right]-\frac{\delta}{4^{n}}\|t\|_{\infty} \\
& \geq\left|t_{n}\right|\left|\xi_{i}\right|-\frac{3 \delta}{4^{n-1}}\|t\|_{\infty} .
\end{aligned}
$$

Hence, for all $n \in \mathbb{N}$, by condition $\left(2^{\prime}\right)$ above with $k:=M(n-1)+1$, we have

$$
\begin{aligned}
& \text { (A) } \max _{N_{M(n-1)}<i \leq N_{M(n)}}\left|\sigma_{i}\right| \geq \max _{N_{M(n-1)}<i \leq N_{M(n-1)+1}}\left|\sigma_{i}\right| \\
& \geq\left|t_{n}\right| \max _{N_{M(n-1)}<i \leq N_{M(n-1)+1}}\left|\xi_{i}\right|-\frac{3 \delta}{4^{n-1}}\|t\|_{\infty} \\
& \geq\left|t_{n}\right|\left(1-\frac{\delta}{4^{M(n-1)+1+2}}\right)-\frac{3 \delta}{4^{n-1}}\|t\|_{\infty} \\
& \geq\left|t_{n}\right|\left(1-\frac{\delta}{4^{n+2}}\right)-\frac{3 \delta}{4^{n-1}}\|t\|_{\infty} \\
& \geq\left|t_{n}\right|-\frac{\delta}{4^{n-2}}\|t\|_{\infty} .
\end{aligned}
$$

Therefore, since $\delta<4^{-7}$,

$$
\|\sigma\|_{\infty}=\max _{n \in \mathbb{N}} \max _{N_{M(n-1)}<i \leq N_{M(n)}}\left|\sigma_{i}\right| \geq \max _{n \in \mathbb{N}}\left|t_{n}\right|-\frac{1}{2}\|t\|_{\infty}=\frac{1}{2}\|t\|_{\infty} .
$$

Hence $\|t\|_{\infty} \leq 2\|\sigma\|_{\infty}$. Now, returning to inequality ( $)$ above, we see that for all $n \in \mathbb{N}$, 


$$
\begin{aligned}
\max _{N_{M(n-1)}<i \leq N_{M(n)}}\left|\sigma_{i}\right| & \leq \frac{\delta}{4^{n-4}}\|t\|_{\infty}+\max \left\{A_{n}\left|t_{n}\right|, A_{n+1}\left|t_{n+1}\right|\right\} \\
& \leq 2 \frac{\delta}{4^{n-4}}\|\sigma\|_{\infty}+\max \left\{A_{n}\left|t_{n}\right|, A_{n+1}\left|t_{n+1}\right|\right\} .
\end{aligned}
$$

Since $\sigma \in c_{0}$, there exists $n_{0} \in \mathbb{N}$ so that

$$
\|\sigma\|_{\infty}=\max _{N_{M\left(n_{0}-1\right)}<i \leq N_{M\left(n_{0}\right)}}\left|\sigma_{i}\right| \text {. }
$$

Then we have

$$
\|\sigma\|_{\infty} \leq 2 \frac{\delta}{4^{n_{0}-4}}\|\sigma\|_{\infty}+\max \left\{A_{n_{0}}\left|t_{n_{0}}\right|, A_{n_{0}+1}\left|t_{n_{0}+1}\right|\right\}
$$

Therefore,

$$
\begin{aligned}
\|\sigma\|_{\infty} & \leq\left[1-\frac{2 \delta}{4^{n_{0}-4}}\right]^{-1} \max \left\{A_{n_{0}}\left|t_{n_{0}}\right|, A_{n_{0}+1}\left|t_{n_{0}+1}\right|\right\} \\
& \leq \max \left\{A_{n_{0}}\left[1-\frac{2 \delta}{4^{n_{0}-5}}\right]^{-1}\left|t_{n_{0}}\right|, A_{n_{0}+1}\left[1-\frac{2 \delta}{4^{n_{0}-4}}\right]^{-1}\left|t_{n_{0}+1}\right|\right\} \\
& \leq \max _{n \in \mathbb{N}}\left\{A_{n}\left[1-\frac{2 \delta}{4^{n-5}}\right]^{-1}\left|t_{n}\right|\right\}=\max _{n \in \mathbb{N}}\left(1+\alpha_{n}\right)\left|t_{n}\right|,
\end{aligned}
$$

where $\alpha_{n}:=A_{n}\left[1-2 \delta / 4^{n-5}\right]^{-1}-1$ for all $n \in \mathbb{N}$. Now $A_{n}=1$ for all $n \geq 2$; hence

$$
\alpha_{n}=\left[1-\frac{2 \delta}{4^{n-5}}\right]^{-1}-1=\frac{2 \delta}{4^{n-5}}\left[1-\frac{2 \delta}{4^{n-5}}\right]^{-1}<\frac{2 \delta}{4^{n-6}} .
$$

So, we have an upper asymptotically isometric $c_{0}$ estimate for $\|\sigma\|_{\infty}$.

To get a corresponding lower estimate, let us return to inequality above. We have

$$
\max _{N_{M(n-1)}<i \leq N_{M(n)}}\left|\sigma_{i}\right| \geq\left|t_{n}\right|-\frac{\delta}{4^{n-2}}\|t\|_{\infty} \geq\left|t_{n}\right|-\frac{2 \delta}{4^{n-2}}\|\sigma\|_{\infty} .
$$

Hence, for all $n \in \mathbb{N}$,

$$
\begin{aligned}
\left|t_{n}\right| & \leq \max _{N_{M(n-1)}<i \leq N_{M(n)}}\left|\sigma_{i}\right|+\frac{2 \delta}{4^{n-2}}\|\sigma\|_{\infty} \\
& \leq\|\sigma\|_{\infty}+\frac{2 \delta}{4^{n-2}}\|\sigma\|_{\infty}=\left(1+\frac{2 \delta}{4^{n-2}}\right)\|\sigma\|_{\infty} ;
\end{aligned}
$$

and so $\left[1+2 \delta / 4^{n-2}\right]^{-1}\left|t_{n}\right| \leq\|\sigma\|_{\infty}$ for all $n \in \mathbb{N}$. Therefore,

$$
\max _{n \in \mathbb{N}}\left(\frac{1}{1+2 \delta / 4^{n-2}}\right)\left|t_{n}\right| \leq\|\sigma\|_{\infty} .
$$

Finally, let $\varepsilon_{n}:=\max \left\{\alpha_{n}, 2 \delta / 4^{n-2}\right\}$ for all $n \in \mathbb{N}$. Then we see that

$$
\max _{n \in \mathbb{N}}\left(\frac{1}{1+\varepsilon_{n}}\right)\left|t_{n}\right| \leq\|\sigma\|_{\infty} \leq \max _{n \in \mathbb{N}}\left(1+\varepsilon_{n}\right)\left|t_{n}\right|,
$$


which means that

$$
\max _{n \in \mathbb{N}}\left(\frac{1}{1+\varepsilon_{n}}\right)\left|t_{n}\right| \leq\left\|\sum_{k=1}^{\infty} t_{k} w_{k}\right\| \leq \max _{n \in \mathbb{N}}\left(1+\varepsilon_{n}\right)\left|t_{n}\right|,
$$

where each $w_{k}:=y_{k}-y_{k-1}$. The proof is now complete since $\left(\varepsilon_{n}\right)$ is a null sequence in $(0, \infty)$. Indeed, for all $n \geq 2, \varepsilon_{n}<2 \delta / 4^{n-6}<2 \cdot 4^{-7} / 4^{n-6}=$ $2^{-1} 4^{-n}$. So we may use the remark preceding this theorem to conclude that $\left(y_{n}\right)_{n \in \mathbb{N}}$ is an asymptotically isometric $c_{0}$-summing basic sequence in $K$. Next, we can apply Theorem 2 directly to find that there exists a nonempty, closed, convex subset $C$ of $K$ and a contractive, affine mapping $T$ on $C$ such that $T$ is fixed point free.

We are now ready to state some consequences of this last theorem. Our first corollary follows from Theorem 4 and Maurey's theorem [9].

Corollary 5. Let $K$ be a closed, bounded, convex subset of $\left(c_{0},\|\cdot\|_{\infty}\right)$. Then $K$ is weakly compact if and only if every closed convex nonempty subset of $K$ has the fixed point property.

Corollary 5 can be extended to the space of convergent sequences, $\left(c,\|\cdot\|_{\infty}\right)$. We first need to prove the following lemma, which may be of independent interest. While we state and prove the lemma with the assumption that the underlying field is $\mathbb{R}$, the result is also true with $\mathbb{C}$ as the underlying field.

Lemma 6. Let $(X,\|\cdot\|)$ be a Banach space over $\mathbb{R}$ and let $K$ be a closed, bounded, convex subset of $X$. Fix a continuous linear functional $\pi \in X^{*}$. Then $K$ is weakly compact if and only if $\pi^{-1}\{a\} \cap K$ is weakly compact for every $a \in \mathbb{R}$.

Proof. Assume $K$ is weakly compact. Fix $a \in \mathbb{R}$. Since $\pi \in X^{*}$, we see that $\pi^{-1}\{a\}$ is norm closed and convex. Thus, $\pi^{-1}\{a\}$ is weakly closed; and so $\pi^{-1}\{a\} \cap K$ is weakly compact.

Conversely, suppose that $\pi^{-1}\{a\} \cap K$ is weakly compact for every $a \in \mathbb{R}$. Let $\varepsilon>0$ be given and let $d:=\operatorname{diam}(K)$. Since $K$ is bounded and convex, $\pi(K)$ is a bounded interval in $\mathbb{R}$. Let $m:=\inf \pi(K)$ and $M:=\sup \pi(K)$. If $M=m$, then $K=\pi^{-1}\{m\} \cap K$ and so $K$ is weakly compact. If $M-m>0$, then $d>0$. Choose $N \in \mathbb{N}$ with $N>1$ so that $d /(N-1)<\varepsilon$. Partition the interval $[m, M]$ into $2 N$ intervals each of length $\delta=(M-m) /(2 N)$. Now define

$$
K_{\varepsilon}:=\bigcup_{j=0}^{2 N}\left(\pi^{-1}\{m+j \delta\} \cap K\right) .
$$

Note that $K_{\varepsilon}$ is weakly compact. We will prove that $K \subseteq K_{\varepsilon}+\varepsilon B_{X}$, where $B_{X}$ is the closed unit ball of $(X,\|\cdot\|)$. By a lemma of Grothendieck [3, Lemma 2, page 227], this is sufficient to prove that $K$ is weakly compact. 
Let $x \in K$. Then $m \leq \pi(x) \leq M$. We assume that $\pi(x) \geq(m+M) / 2$ $=m+N \delta$. (The case where $\pi(x) \leq(m+M) / 2$ is handled in a similar manner.) Define $j_{0}:=\max \{j: m+j \delta \leq \pi(x)\}$. Then $j_{0} \geq N$. Since $m+\delta \in$ $\pi(K)$, we can choose $w \in \pi^{-1}\{m+\delta\} \cap K$. Define

$$
z:=s w+(1-s) x, \quad \text { where } \quad s:=\frac{\pi(x)-\left(m+j_{0} \delta\right)}{\pi(x)-(m+\delta)} \in[0,1) .
$$

It is easy to check that $\pi(z)=m+j_{0} \delta$ and also $z \in K$, since $K$ is convex. Thus $z \in K_{\varepsilon}$. Finally, we note that

$$
\|z-x\|=s\|w-x\| \leq s d \leq \frac{\delta}{N \delta-\delta} d<\varepsilon .
$$

Thus $x \in K_{\varepsilon}+\varepsilon B_{X}$; and so $K \subseteq K_{\varepsilon}+\varepsilon B_{X}$.

REMARK. Let us sketch how the above proof goes in the case of complex scalars. Clearly, if $K$ is weakly compact, then so is $\pi^{-1}\{a\} \cap K$ for every $a \in \mathbb{C}$.

On the other hand, suppose that $\pi^{-1}\{a\} \cap K$ is weakly compact for every $a \in \mathbb{C}$. Here $\pi$ maps $X$ into $\mathbb{C}$, and $\Re \pi$ and $\Im \pi$ are continuous real-linear functionals on $(X,\|\cdot\|)$. For each $t \in \mathbb{R}$,

$$
L_{t}:=(\Re \pi)^{-1}\{t\} \cap K=\bigcup_{u \in \mathbb{R}}\left(L_{t} \cap(\Im \pi)^{-1}\{u\}\right) .
$$

But, for each $u \in \mathbb{R}$,

$$
L_{t} \cap(\Im \pi)^{-1}\{u\}=\pi^{-1}\{t+i u\} \cap K,
$$

which, by hypothesis, is a weakly compact subset of $X$.

Since convexity is a real-linear property, our real scalars proof above, applied with $\Im \pi$ in place of $\pi$, shows that $L_{t}:=(\Re \pi)^{-1}\{t\} \cap K$ is weakly compact. (Note that Grothendieck's weak compactness criterion applies to both real and complex Banach spaces.)

Now, $t \in \mathbb{R}$ is arbitrary. So, repeating the preceding argument for $\Re \pi$ instead of $\Im \pi$, we conclude that $K$ is weakly compact.

REMARK. The statement of Lemma 6 is also true if weak compactness is replaced by norm compactness.

Remark. We note that by the Kreŭn-Shmul'yan theorem, Lemma 6 implies that whenever $K$ is a weakly closed, bounded subset of a Banach space $X$ and $\pi \in X^{*}$, then $K$ is weakly compact if and only if for all scalars $a$, the intersection of $\pi^{-1}\{a\}$ with the closed convex hull of $K$ is weakly compact.

However, the hypothesis in Lemma 6 that $K$ is convex cannot be removed. Indeed, the hypothesis " $K$ is closed and convex" cannot be replaced by " $K$ is weakly closed", as the following example shows. Let $X:=c$ and define the map $\pi: c \rightarrow \mathbb{R}$ by $\pi\left(x_{1}, x_{2}, \ldots\right):=\lim _{n} x_{n}$, for each $\left(x_{1}, x_{2}, \ldots\right) \in c$. Define 


$$
z^{(k)}:=\left(1,0,1^{2}, 0, \ldots, 1^{k}, 0,1 / k, 1 / k, \ldots\right) \quad \text { for all } k \in \mathbb{N} .
$$

Clearly $K:=\left\{z^{(k)}: k \in \mathbb{N}\right\}$ is a bounded subset of $c$, and it is easy to check that $K$ is weakly closed. Moreover, $\pi^{-1}\{1 / k\} \cap K=\left\{z^{(k)}\right\}$ for all $k \in \mathbb{N}$ and $\pi^{-1}\{a\} \cap K=\emptyset$ for all other real numbers $a$. So, $\pi^{-1}\{a\} \cap K$ is weakly compact for all $a \in \mathbb{R}$.

On the other hand, $K$ is not weakly compact. To see this, use Banach's criterion for weak convergence in $c$ [1, IX, pp. 136-137]. Since $\left(z^{(k)}\right)_{k \in \mathbb{N}}$ converges coordinatewise to $q:=(1,0,1,0,1,0, \ldots)$, which is not in $c$, it follows that $K$ cannot be weakly compact.

Corollary 7. Let $K$ be a closed, bounded, convex subset of $\left(c,\|\cdot\|_{\infty}\right)$. Then $K$ is weakly compact if and only if every closed, convex, nonempty subset of $K$ has the fixed point property.

Proof. If $K$ is a closed, bounded, convex subset of $\left(c,\|\cdot\|_{\infty}\right)$ which is weakly compact, then all of its closed (bounded) convex, nonempty subsets have the fixed point property by a result of Borwein and Sims [2].

Conversely, if $K$ is closed, bounded, convex and non-weakly compact in $\left(c,\|\cdot\|_{\infty}\right)$, then by Lemma 6 , there exists $a \in \mathbb{R}$ so that $\pi^{-1}\{a\} \cap K$ is non-weakly compact, where $\pi \in c^{*}$ is defined by $\pi\left(x_{1}, x_{2}, \ldots\right):=\lim _{n} x_{n}$ for each $\left(x_{1}, x_{2}, \ldots\right) \in c$.

Define $U: \pi^{-1}\{a\} \rightarrow c_{0}$ by $U(x):=\left(x_{n}-a\right)_{n}$ for each $x=\left(x_{n}\right)_{n} \in$ $\pi^{-1}\{a\}$. It is easily seen that $U$ is an affine map from $\left(\pi^{-1}\{a\},\|\cdot\|_{\infty}\right)$ onto $\left(c_{0},\|\cdot\|_{\infty}\right)$ and $\|U(x)-U(y)\|_{\infty}=\|x-y\|_{\infty}$ for all $x, y \in \pi^{-1}\{a\}$. Moreover, it is easy to check that $U$ is a $\left(\pi^{-1}\{a\}, \sigma\left(c, \ell_{1}\right)\right)$ to $\left(c_{0}, \sigma\left(c_{0}, \ell_{1}\right)\right)$ homeomorphism. Here $\sigma\left(c, \ell_{1}\right)$ is the weak topology on $c$. (See, for example, [1, IV, pp. 65-67].)

Therefore the non-weakly compact, closed, bounded, convex subset $\pi^{-1}\{a\} \cap K$ in $\pi^{-1}\{a\}$ corresponds isometrically to the non-weakly compact, closed, bounded, convex subset $U\left(\pi^{-1}\{a\} \cap K\right)$ in $c_{0}$. By Theorem 4, $U\left(\pi^{-1}\{a\} \cap K\right)$ contains a subset $C$ which is closed, bounded and convex such that there is a nonexpansive mapping $V: C \rightarrow C$ that has no fixed point. It follows that $U^{-1}(C)$ is a closed, bounded, convex subset of $\pi^{-1}\{a\} \cap K$ and the mapping $U^{-1} V U$ is a nonexpansive mapping on $U^{-1}(C)$ which fails to have a fixed point in $U^{-1}(C)$.

REMARK. By the Remark after Lemma 6, the previous corollary is also true if we replace the field $\mathbb{R}$ everywhere by $\mathbb{C}$.

Our next corollary follows from the Eberlein-Shmul'yan theorem and the proof of Theorem 4 .

Corollary 8. Let $K$ be a subset of $\left(c_{0},\|\cdot\|_{\infty}\right)$. Then $K$ is relatively weakly compact if and only if $K$ is bounded and the convex hull of $K$, 
$\operatorname{co}(K)$, does not contain an L-scaled asymptotically isometric $c_{0}$-summing basic sequence for any $L>0$.

Corollary 8, as stated, is an isometric characterization of relative weak compactness in $c_{0}$. However, since relative weak compactness is an isomorphic property, Corollary 8 would be more useful if it was formulated in an isomorphic form. The isomorphic form of this result is that a subset $K$ of $c_{0}$ is relatively weakly compact if and only if it is bounded and its convex hull, $\operatorname{co}(K)$, does not contain a sequence equivalent to the summing basis of $c_{0}$. This result would be even more useful if the dependence on the convex hull of $K$ were removed. This can be done by suitably modifying the proof of Theorem 4.

THEOREM 9. Let $K$ be a subset of $c_{0}$. Then $K$ is relatively weakly compact if and only if $K$ is bounded and $K$ does not contain a sequence equivalent to the summing basis of $c_{0}$.

Proof. If $K$ is relatively weakly compact, then $K$ is bounded. Now suppose, to get a contradiction, that $K$ contains a sequence $\left(x_{n}\right)_{n \in \mathbb{N}}$ equivalent to the summing basis of $c_{0}$. It is easy to check that $x_{n} \rightarrow z$ for some $z \in \ell_{\infty} \backslash c_{0}$ with respect to the weak ${ }^{*}=\sigma\left(\ell_{\infty}, \ell_{1}\right)$ topology. This contradicts the fact that $K$ is relatively weakly compact.

The second part of the proof of Theorem 9 is very similar to the proof of Theorem 4. If $K$ is a bounded subset of $c_{0}$ that is not relatively weakly compact then, just as in the proof of Theorem 4, we construct a sequence $\left(x_{n}\right)_{n}$ in $K$ and an element $x$ in $\ell_{\infty} \backslash c_{0}$ satisfying conditions $\left(1^{\prime}\right)-\left(4^{\prime}\right)$. Now instead of averaging the $x_{n}$ 's (which we cannot do unless $K$ is convex), we show that the subsequence consisting of the odd terms, $\left(x_{2 n-1}\right)$, is equivalent to the summing basis of $c_{0}$. The details of the proof are technical, as they are in Theorem 4, but the proof is so similar to the proof of Theorem 4 that we will leave it to the reader to fill in the details.

Remark. Theorem 9 is similar to [4, Proposition 3.1(c)] (which is stated for the case where $K$ is also assumed to be convex).

REMARK. One should compare Theorem 9 to [11, III.C.9], where the following characterization of relative weak compactness in $\ell_{1}$ is stated. A subset $H$ of $\ell_{1}$ is relatively weakly compact if and only if $H$ is bounded and $H$ does not contain a sequence equivalent to the usual unit vector basis of $\ell_{1}$. (Of course, for $\ell_{1}$, via the Schur property, these conditions are also equivalent to: $H$ is relatively norm compact.)

\section{References}

[1] S. Banach, Théorie des opérations linéaires, Chelsea, New York, 2nd ed., 1978. 
[2] J. M. Borwein and B. Sims, Nonexpansive mappings on Banach lattices and related topics, Houston J. Math. 10 (1984), 339-356.

[3] J. Diestel, Sequences and Series in Banach Spaces, Springer, New York, 1984.

[4] T. Domínguez Benavides, M. A. Japón Pineda and S. Prus, Weak compactness and fixed point property for affine mappings, J. Funct. Anal., to appear.

[5] P. N. Dowling, C. J. Lennard and B. Turett, Asymptotically isometric copies of $c_{0}$ in Banach spaces, J. Math. Anal. Appl. 219 (1998), 377-391.

[6] R. Haydon, E. Odell and Y. Sternfeld, A fixed point theorem for a class of star-shaped sets in $c_{0}$, Israel J. Math. 38 (1981), 75-81.

[7] P. K. Lin, Unconditional bases and fixed points of nonexpansive mappings, Pacific J. Math. 116 (1985), 69-76.

[8] E. Llorens-Fuster and B. Sims, The fixed point property in $c_{0}$, Canad. Math. Bull. 41 (1998), 413-422.

[9] B. Maurey, Points fixes des contractions de certains faiblement compacts de $L^{1}$, in: Seminar on Functional Analysis, 1980-1981, École Polytech., Palaiseau, 1981, Exp. No. VIII, 19 pp.

[10] E. Odell and Y. Sternfeld, A fixed point theorem in $c_{0}$, Pacific J. Math. 95 (1981), 161-177.

[11] P. Wojtaszczyk, Banach Spaces for Analysts, Cambridge Univ. Press, Cambridge, 1991.

Department of Mathematics and Statistics

Miami University

Oxford, OH 45056, U.S.A.

E-mail: dowlinpn@muohio.edu

Department of Mathematics and Statistics

Oakland University

Rochester, MI 48309, U.S.A.

E-mail: turett@oakland.edu
Department of Mathematics University of Pittsburgh Pittsburgh, PA 15260, U.S.A.

E-mail: lennard+@pitt.edu

Received April 8, 2002

Revised version August 9, 2002 